\title{
Ipsilateral Recurrence of Nonarteritic Anterior Ischemic Optic Neuropathy: A Case Report
}

\author{
Daniel Brigham, OD, FAAO \\ Carl Vinson Veterans Affairs \\ Medical Center
}

\begin{abstract}
Nonarteritic anterior ischemic optic neuropathy (NA-AION) is a multifactorial disease that is the most common type of ischemic optic neuropathy. NA-AION develops in one eye and can later develop in the fellow eye. The ipsilateral recurrence of NA-AION is rare and multiple theories have been proposed to explain it. This case report describes a patient with unilateral recurrence of NA-AION, explores the proposed etiologies, and emphasizes visual symptoms following its resolution.
\end{abstract}

\section{KEY WORDS:}

optic nerve edema, recurrent, nonarteritic ischemic optic neuropathy, contrast sensitivity, peripapillary retinal nerve fiber layer ( $\mathrm{pRNFL}$ )

\section{INTRODUCTION}

Nonarteritic anterior ischemic optic neuropathy (NA-AION) represents $95 \%$ of all anterior ischemic optic neuropathies. ${ }^{1}$ It is one of the most visually debilitating diseases in patients over the age of 50, with an estimated annual incidence in the United States of 2.3 to 10.2 per 100,000 population, which equates to approximately 6,000 new cases annually. ${ }^{1}$ NA-AION affects a significantly higher proportion of Caucasians compared to other races, with a mean age of onset of 66 years. ${ }^{1}$ Visual acuity at initial presentation is variable; $49 \%$ of patients have an initial visual acuity of $\geq 20 / 64$, while $23 \%$ to $34 \%$ have an initial visual acuity of $\leq 20 / 200{ }^{1,2}$ Further, $38 \%$ have minimal to mild visual field defects and $43 \%$ have marked to severe defects. ${ }^{2}$

The pathogenesis of NA-AION, albeit controversial, has been proposed to involve one of two mechanisms. The most commonly accepted mechanism is transient nonperfusion or hypoperfusion of the optic nerve head $(\mathrm{ONH}){ }^{3}$ The second proposed cause is embolic occlusion of the arterioles supplying the $\mathrm{ONH} .{ }^{3}$ When considering the former, two main categories of risk factors exist: predisposing risk factors (differentiated into systemic risk factors and local ONH risk factors) and precipitating risk factors. ${ }^{4}$

The occurrence of NA-AION in the contralateral eye is possible, and approximately $25 \%$ of patients suffer involvement in the fellow eye within 3 years of that in the primary eye. ${ }^{5}$ However, recurrence in the same eye is rare. In a study including 594 consecutive patients with NA-AION, only $6 \%$ developed NA-AION a second time in the ipsilateral eye. ${ }^{6}$ The following case report highlights an ipsilateral recurrence of NA-AION and expands on the proposed etiologies of NA-AION, with an emphasis on ipsilateral recurrence. Additionally, common visual complaints in NA-AION are discussed.

\section{CASE REPORT}

In 2017, a 76-year-old Caucasian man presented to our clinic with complaints of new-onset intermittent brown spots of different shapes and sizes in the central vision of his right eye. These spots began three days prior and 
were noted a few times per day. He additionally described a "glow" on the right side of his vision with an associated dull ache behind his right eye. He denied headache, scalp tenderness, jaw claudication, fever, weight loss, Uhthoff"s phenomenon, numbness, and weakness.

His medical history was notable for hypertension, hyperlipidemia, coronary artery disease, venous insufficiency, gastroesophageal reflux, insomnia, and left-sided Bell's Palsy 20 years previously. Current medications were citalopram (Celexa), furosemide (Lasix), lisinopril (Zestril), metoprolol (Lopressor), nitroglycerin (Nitrostat), omeprazole (Prilosec), simvastatin (Zocor), and 325mg aspirin (Bayer). He denied use of phosphodiesterase-5 inhibitors, tobacco, alcohol, or recreational drugs.

His past ocular history was significant for NA-AION OS in 2007 and visually insignificant cataracts OU. He denied any history of eye surgeries, eye trauma, or family history of ocular disease.

His blood pressure was 142/79 $\mathrm{mm} \mathrm{Hg}$ at 2:30pm.

His best-corrected visual acuity was $20 / 20^{-1}$ OD and finger-counting at 0.3 meters OS. He had normal ocular motility without pain or double vision. Pupils were equal and round with a $3^{+}$left relative afferent pupillary defect. Confrontation visual fields revealed an inferior nasal restriction OD and complete inferior restriction OS. Color vision testing with HRR pseudoisochromatic plates was intact OD (6/6 plates) and could not be performed OS due to his profoundly reduced acuity. The right and left superficial temporal arteries were easily palpable and nontender. Slit-lamp biomicroscopy was unremarkable, with the exception of $2^{+}$nuclear sclerotic cataracts OU. Intraocular pressures were $15 \mathrm{~mm} \mathrm{Hg}$ OU at 2:00pm as measured with Goldmann applanation tonometry.

Dilated fundus examination showed superior ONH edema OD with a solitary albeit large retinal nerve fiber layer hemorrhage at the nasal margin (Figure 1). The left ONH had diffuse pallor with distinct margins (Figure 2). For reference, the cup to disc ratios were previously determined to be 0.3 round OD and 0.25 round OS with an optic disc size of $1.6 \mathrm{~mm}$ OU. The remainder of the fundus examination was unremarkable.

Additional testing was pursued including spectral domain optical coherence tomography (SD-OCT) imaging of the peripapillary retinal nerve fiber layer (pRNFL) (Figure 3). This was obtained with a Heidelberg Spectralis SD-OCT (Heidelberg Engineering, Inc., Heidelberg, Germany) and revealed hemispheric superior pRNFL edema with normal inferior pRNFL thickness.

Figure 1: Fundus photograph taken at the initial visit OD. Superior ONH edema with a nasal flame shaped hemorrhage is evident.

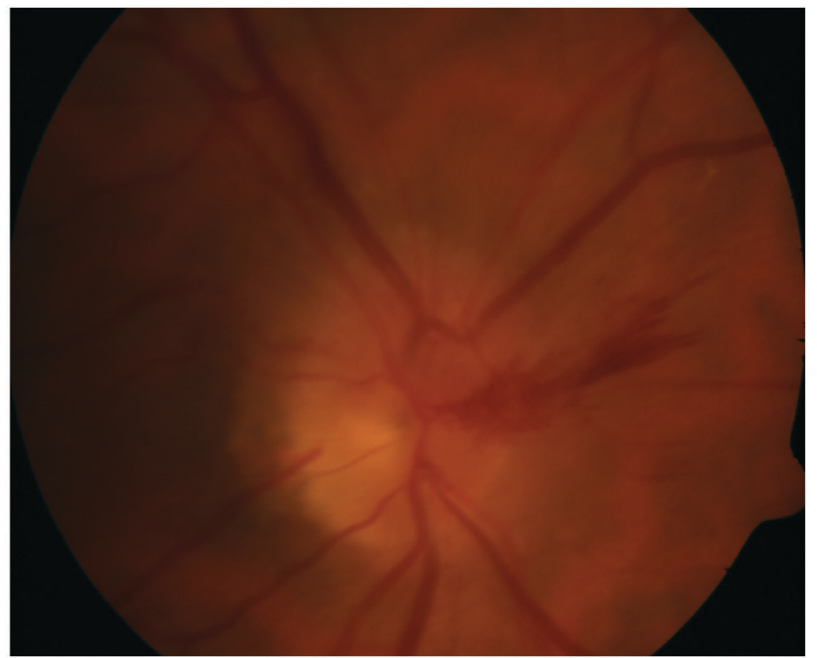


Figure 2: Fundus photograph OS showing diffused ONH pallor.

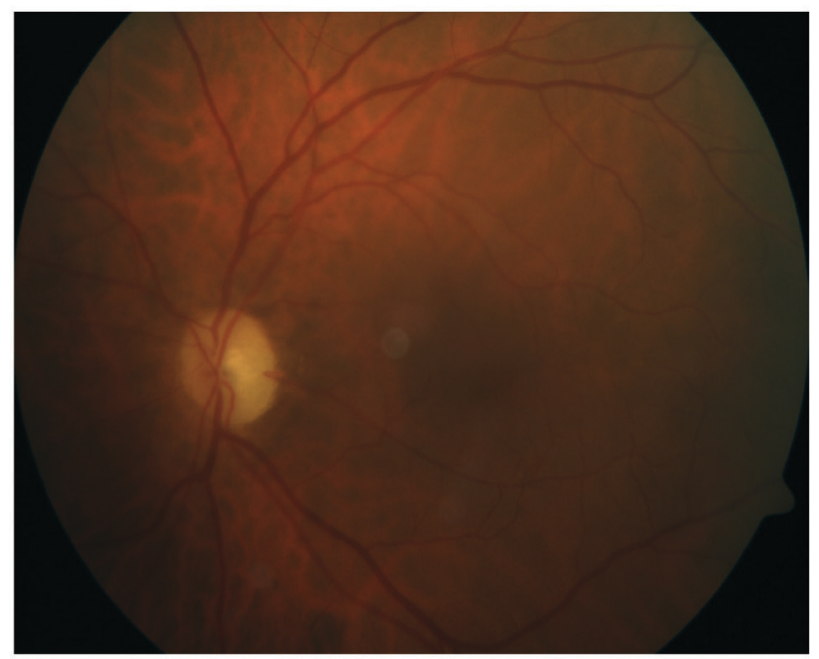

Figure 3: OCT-RNFL OD acquired at the initial visit shows hemispheric superior thickening.
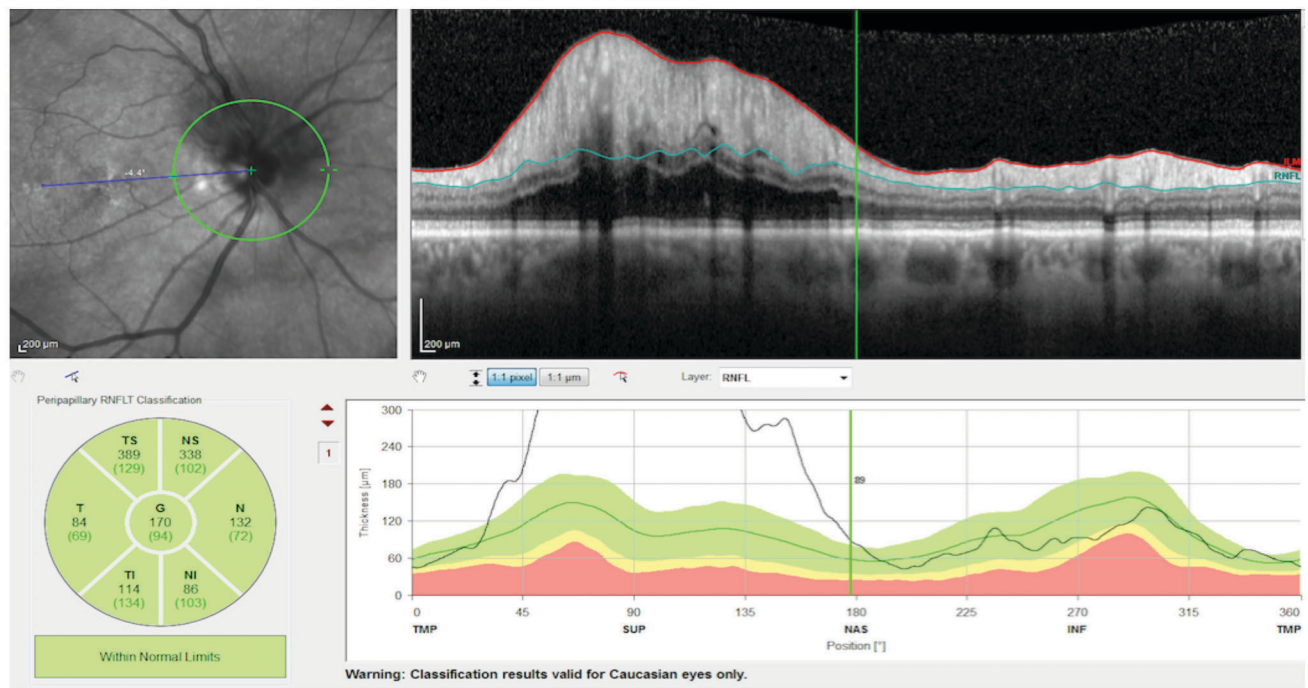

Differential diagnoses considered for this patient included other potential causes of ONH edema, as well as possible causes of $\mathrm{ONH}$ edema in one eye and $\mathrm{ONH}$ pallor in the fellow eye. These included demyelinating (multiple sclerosis), inflammatory (sarcoidosis, systemic lupus erythematosus), compressive (optic nerve sheath meningioma, Foster Kennedy syndrome), infectious (tuberculosis, bartonella, syphilis, Lyme), neoplastic (lymphoma, leukemia, glioma), and vascular (arteritic anterior ischemic optic neuropathy (A-AION), NA-AION) etiologies.?

Despite the presumptive diagnosis of NA-AION, same-day lab-testing was pursued and included a comprehensive metabolic panel (CMP), complete blood count (CBC), C-reactive protein (CRP), erythrocyte sedimentation rate (ESR), and glycated hemoglobin (HBAlc). All the results were within normal reference ranges for the patient's age and existing comorbidities: specifically, an ESR of $7 \mathrm{~mm} / \mathrm{h}$ and a CRP of $<5.0 \mathrm{mg} / \mathrm{L}$ helped rule out A-AION secondary to giant cell arteritis. A previous sleep study showed no sleep apnea. 
Due to the clinical presentation and normal lab-testing results, a diagnosis of NA-AION was made. The patient was educated on the findings and the course of the disease, and on better control of potential risks factors for NA-AION, including his underlying systemic diseases. Additionally, the timing of the use of his systemic anti-hypertensive medications was discussed in detail. The patient denied use of his systemic anti-hypertensive medications within 3 to 4 hours of falling asleep despite altered sleeping patterns due to insomnia. A note was sent to his primary care provider with today's exam findings. The patient was directed to return to the clinic in one week for ongoing care, including dilated fundus exam, SD-OCT, and Humphrey visual field 24-2 testing (HVF 24-2).

The patient presented one week later with no new visual or systemic complaints. His best-corrected visual acuity was $20 / 20^{-2}$ OD and finger-counting at 0.3 meters OS. The remaining preliminary tests were stable and consistent with the previous visit. Dilated fundus examination displayed a similar ONH appearance OD (Figure 4), although SD-OCT revealed a slight decrease in the superior pRNFL thickness consistent with resolving edema OD (Figure 5). HVF 24-2 testing could not be performed at this visit due to malfunctioning equipment. The patient was educated on the course of NA-AION and directed to return in one month for HVF 24-2 testing.

Figure 4: Fundus photograph OD one week after initial presentation.

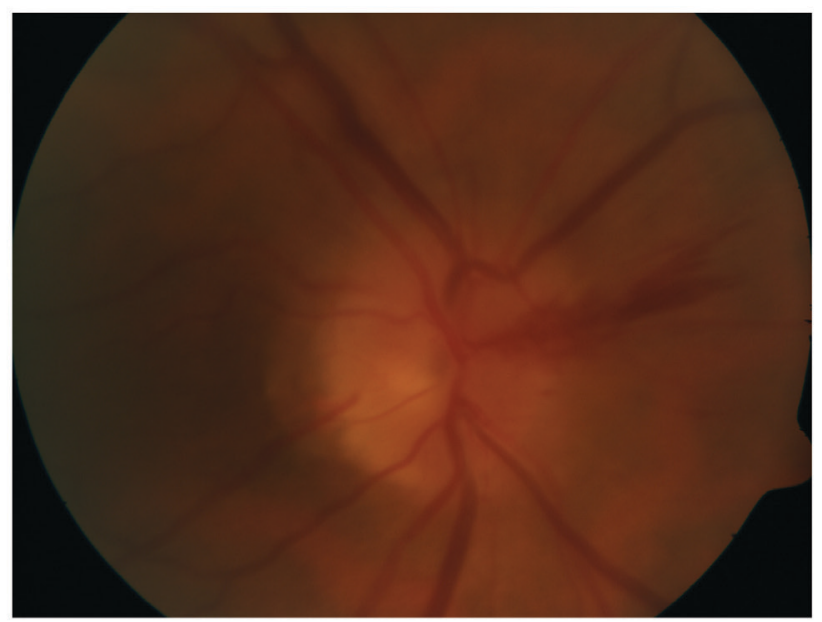

Figure 5: OCT-RNFL OD acquired one week after initial presentation shows improvement in hemispheric superior thickening with mild thickening nasal.

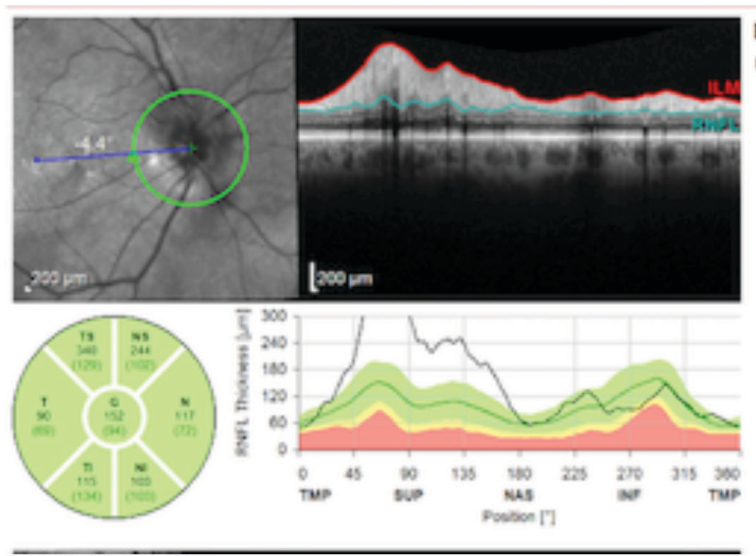

Follow.Up \#1 Mar/2/2017

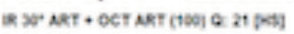

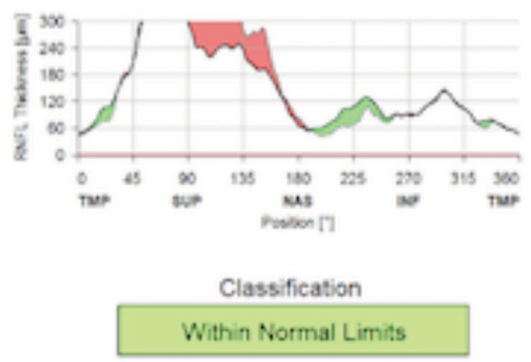


At the 10th week follow-up visit (the patient missed his fifth week follow-up), the patient had new complaints of reduced central visual acuity in his right eye of three days' duration. Additionally, he now noticed changes in his ability to distinguish shades and had an increase in light sensitivity. His best corrected visual acuity was reduced to 20/30- OD. Mars Letter Contrast Sensitivity testing of the right eye showed a severe contrast sensitivity loss, with a log value of 0.56 .

Dilated fundus examination demonstrated near-complete resolution of the superior ONH edema and RNFL hemorrhage, however inferior ONH edema was now present (Figure 6). SD-OCT confirmed this presentation with thinning of the pRNFL in the superior temporal sector and thickening in the inferior hemisphere (Figure 7). An HVF 24-2 test using a SITA Standard strategy OD was reliable with fixation losses of $1 / 18$, false-positive errors of $0 \%$, false-negative errors of $20 \%$, and excellent gaze-tracking. The mean deviation was $-16.50 \mathrm{~dB}$ and the visual field index was $46 \%$. There were dense inferior and superior central defects consistent with both previous superior and current inferior ONH involvement, respectively (Figure 8). HVF 24-2 testing was not attempted OS due to profound vision loss.

Figure 6: Fundus photograph OD taken six weeks after initial presentation now shows inferior ONH edema.

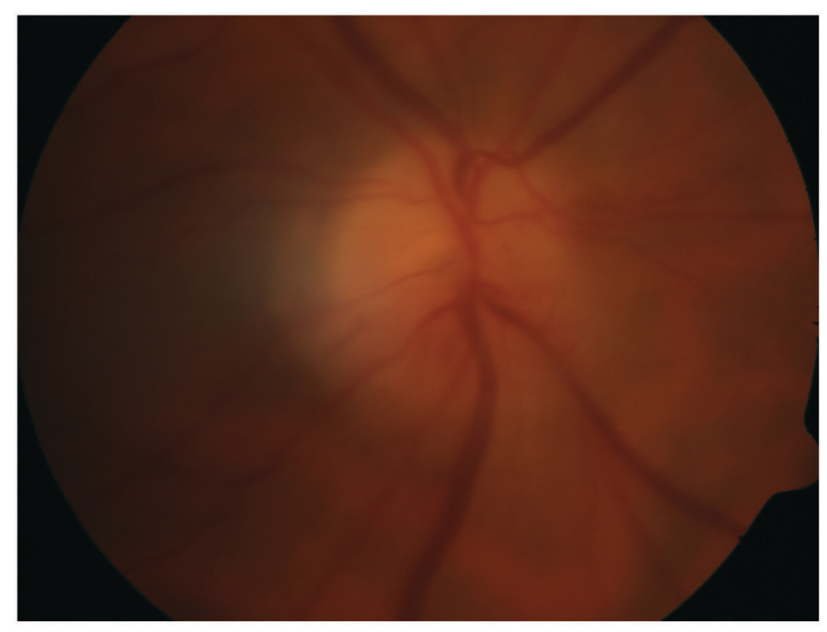

Figure 7: OCT-RNFL OD acquired six weeks after initial presentation shows complete resolution of hemispheric superior thickening, superior temporal sector thinning, and new hemispheric inferior thickening.

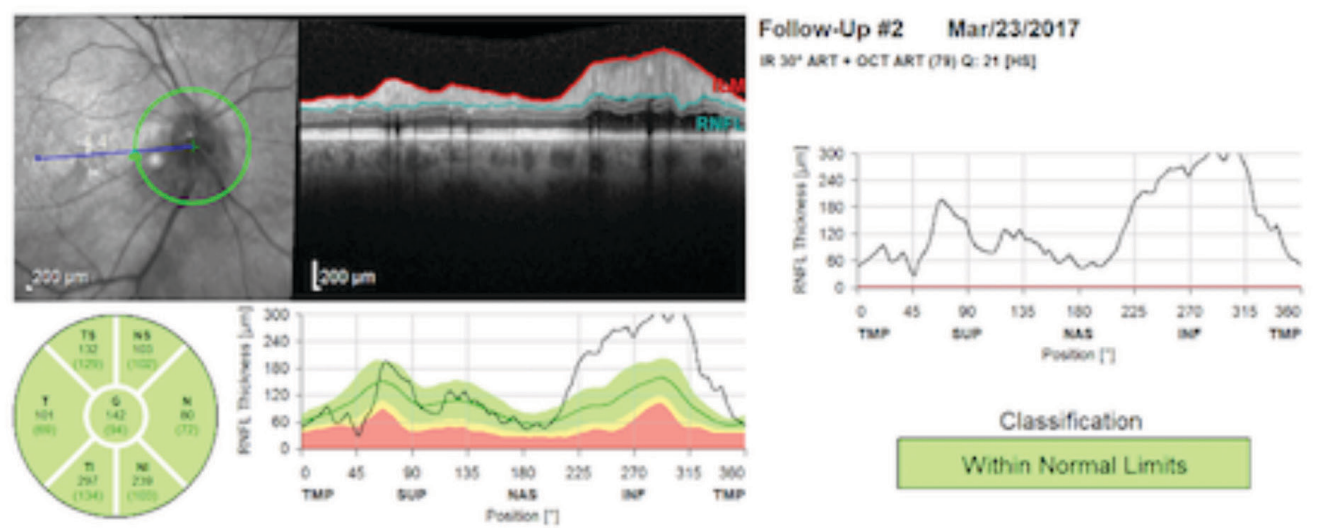


Figure 8: Humphrey visual field 24-2 sita standard testing following the initial and recurrent NA-AION OD.

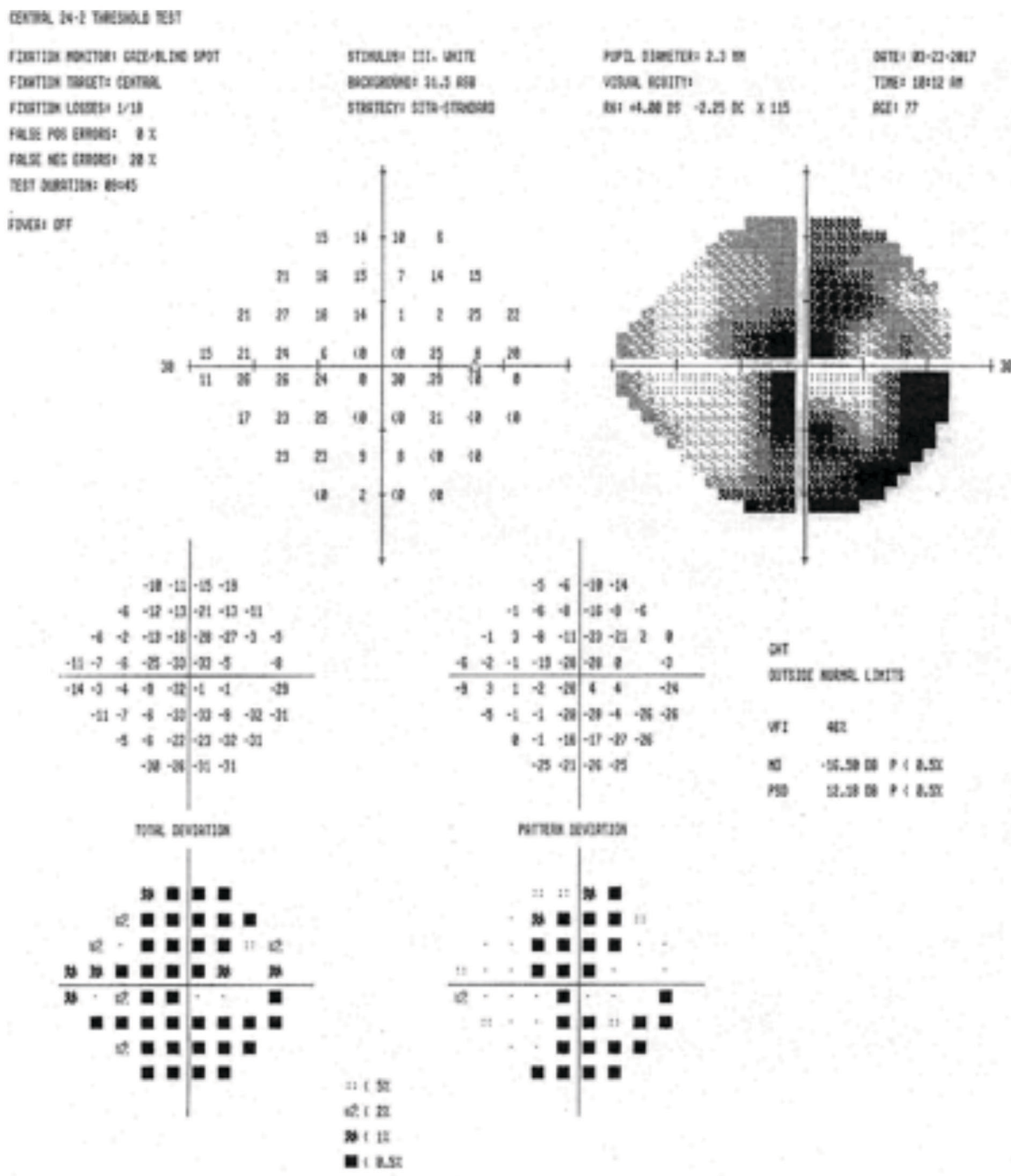

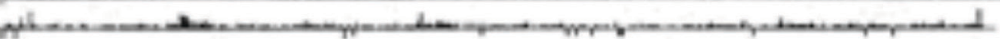

The patient was educated on the rarity of his ipsilateral recurrence of NA-AION. The importance of controlling potential systemic risk factors was reinforced, resulting in the patient admitting to poor adherence with his prescribed medications. Further, he noted that his sleep schedule had changed over the last few weeks secondary to stress and he had begun to use his systemic anti-hypertensive medications closer to falling asleep. 
The patient was scheduled to return in one month for a dilated fundus examination with SD-OCT testing OD and to repeat the HVF 24-2 OD. He was also referred for vision rehabilitation services to help improve his daily activities that had become affected.

One month later (14th week follow-up) there was complete resolution of the ONH edema. The disc was now diffusely pale secondary to the post edema atrophic process (Figure 9). The visual field defect and visual acuity remained unchanged. The patient was to keep his scheduled vision rehabilitation evaluation and return in four months for HVF 10-2 testing. The patient did not return for follow-up examinations beyond his visual rehabilitation care.

Figure 9: Fundus photograph OD taken ten weeks after initial presentation and four weeks after the ipsilateral recurrence shows reduced inferior $\mathrm{ONH}$ edema and increased $\mathrm{ONH}$ pallor.

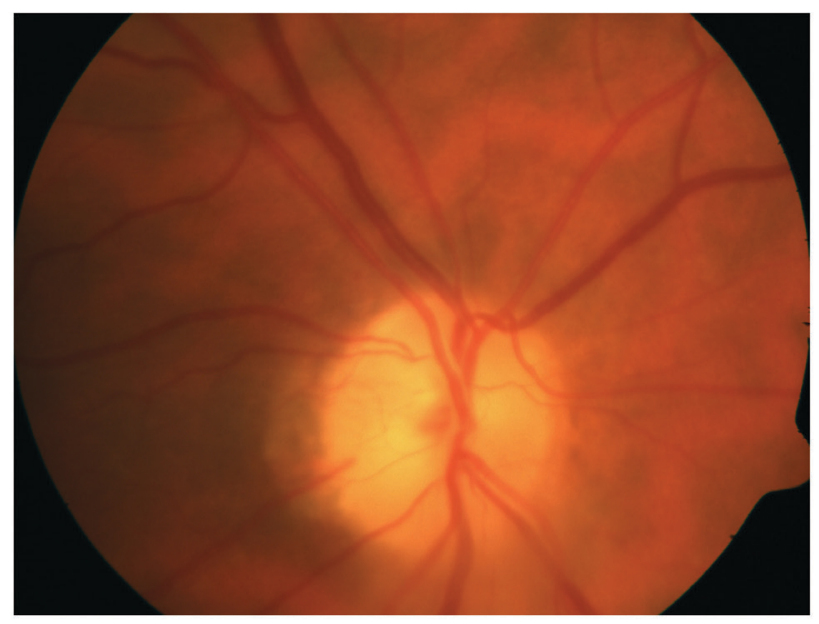

During the visual rehabilitation exam our patient demonstrated a difficulty with contrast sensitivity and photophobia that we were able to improve with the use of a light-amber filter for inside wear and a wraparound dark-amber filter for outside wear. The patient was also provided a handheld video magnifier with built-in contrast enhancement to facilitate reading tasks. A discussion of driving restrictions was not required as the patient ceased driving on his own accord following his first NA-AION occurrence.

DISCUSSION

Non-arteritic anterior ischemic optic neuropathy (NA-AION) is an ischemic disorder of the posterior ciliary artery (PCA) circulation, the main source of blood supply to the optic nerve head $(\mathrm{ONH}){ }^{8-10}$ More recent studies using optical coherence tomography angiography (OCT-A) have shown impaired flow in both the retinal peripapillary capillaries (RPC) and peripapillary choriocapillaris (PCC) following an episode of NA-AION. ${ }^{11,12}$ These areas show a strong correspondence to both functional and structural changes. ${ }^{11}$ Presumably, hypoperfusion of the RPC after an episode of NA-AION results as a consequence of an inciting ischemic event within the PCA circulation. ${ }^{11}$ One proposed explanation for this decrease in RPC perfusion is that RPCs possess unique anatomical features not found in other capillaries: they tend to be longer and straighter, and connect large-caliper arteries to the long venous trunk directly, without anastomosis. $^{12}$ These peculiarities make RPCs more sensitive to changes in blood flow, such as those that occur with ischemic events within the PCA circulation. ${ }^{12}$

The exact pathogenesis of NA-AION remains controversial, but has been proposed to involve one of two processes. Most commonly, transient non-perfusion or hypoperfusion of the ONH is cited. Rarely, NA-AION is attributed to embolic lesions of the arterioles supplying the $\mathrm{ONH} .{ }^{8}$ The former will be discussed below.

Fluorescein angiography (FA) at the onset of NA-AION has shown delayed filling of the peripapillary choroid and its watershed zones (a border between the territories of distribution of any two end arteries) as opposed to a complete lack of filling. ${ }^{13}$ This can be interpreted to mean that these vessels are not absolutely occluded. ${ }^{13}$ Since there is only a transient 
loss of circulation to the $\mathrm{ONH}$, there is usually a much less severe ischemic cascade and resultant ONH damage. This was demonstrated in two large studies, in which approximately $41 \%$ of eyes with NA-AION showed spontaneous visual improvement. ${ }^{1,2}$ It is postulated that this hypoperfusion is a result of the drop in perfusion pressure of the capillaries of the $\mathrm{ONH}$ below the critical autoregulatory range. ${ }^{8}$ Autoregulation is critical in maintaining proper blood flow to a tissue through the dilatation and constriction of blood vessels in response to perfusion pressure and local metabolic demand. However, autoregulation will only operate over a certain range of perfusion pressure and becomes ineffective beyond that range; at this point, blood flow to the $\mathrm{ONH}$ becomes directly correlated to the perfusion pressure. ${ }^{14}$

Several variables, in different combinations, can render the $\mathrm{ONH}$ more susceptible to an ischemic insult. The specific combination of factors that result in NA-AION will be unique to each patient affected, underscoring the notion that NA-AION is multifactorial in nature. ${ }^{8}$ These risk factors can be categorized as either predisposing risk factors (differentiated into systemic risk factors and local $\mathrm{ONH}$ risk factors) or precipitating risk factors. ${ }^{4}$

Predisposing risk factors make the $\mathrm{ONH}$ more susceptible to an ischemic event. Systemic factors include arterial hypertension, diabetes mellitus, ischemic heart disease, sleep apnea, and hyperlipidemia, among others. ${ }^{15-17}$ Several of these risks factors were present in our patient. Local ONH factors may also play a predisposing role, and include absent or small cup in the optic disk (secondary contributing factor) ${ }^{18}$ significantly raised IOP, ${ }^{19}$ optic disk drusen, ${ }^{19}$ and the location of the watershed zone formed by the distribution of the posterior ciliary arteries in relation to the $\mathrm{ONH}{ }^{20}$

The most notable precipitating risk factor that may ultimately act as the final insult to the susceptible nerve, leading to the cascade of ischemia that deprives the $\mathrm{ONH}$, is nocturnal arterial hypotension. ${ }^{21-23}$ It has been documented that $73 \%$ of patients with NA-AION specifically reported that their vision loss was noted upon waking from sleep/nap or when they first used their vision critically. ${ }^{24}$ Furthermore, 24-hour ambulatory blood pressure monitoring has shown many cases of nocturnal arterial hypotension in individuals who developed NA-AION.22,23 A significant relationship between nocturnal arterial hypotension and the use of potent oral anti-hypertensive therapy has been established..$^{22,23}$ In fact, since the advent of these hypotensive treatment agents, there has been a rise in the incidence of NA-AION. ${ }^{8}$ In this case, the patient was taking three potent hypotensive drugs, and admittedly dosed them near bedtime prior to the ipsilateral recurrence.

For patients with NA-AION, the risk of contralateral eye involvement is reported to range from $14.7 \%$ to $25 \%$ over three to five years. ${ }^{5,25}$ However, recurrence in the ipsilateral eye is considered to be rare. It is important to differentiate true ipsilateral recurrence from a progression of NA-AION during the acute stage of the disease.

Optic nerve edema has a characteristic evolutionary pattern in NA-AION. ${ }^{26}$ Initially, a portion of the ONH is edematous, with the remainder of the disc unaffected. After several days, the entire nerve can manifest diffusely generalized edema. Sequentially, the initially involved section of the $\mathrm{ONH}$ will develop pallor in response to the previous ischemic axonal insult. Edema may persist throughout the rest of the $\mathrm{ONH}$. This edema will also gradually resolve, with resultant pallor.

In this case, from initial presentation to one week post presentation, there was some resolution of the superior edema with a subtle increase in the inferior nasal edema (Figure 3 and Figure 5). This migration of edema from its starting point to an originally uninvolved area is not uncommon based on the findings described previously. From one week through 10 weeks post presentation, the superior edema fully resolved, leaving some RNFL thinning. However, significant inferior edema, well beyond what was seen with the initial migration of edema at the one week post presentation, had developed (Figure 5 and Figure 7). This new development of significant inferior ONH edema, coupled with the 7.9 week median average time for complete resolution of ONH edema after acute NA-AION ${ }^{26}$ substantiate that the patient suffered from recurrent ipsilateral NA-AION.

Several hypotheses have been suggested to explain the rarity of ipsilateral recurrence of NA-AION, including the inability of the patient to recognize further visual loss after the initial episode, ${ }^{27}$ shunting of blood from the damaged to the healthy part of the $\mathrm{ONH},{ }^{27}$ and initial destruction of the RNFL in a crowded optic disk providing more space for the surviving RNFL ${ }^{28}$. However, these postulated ideas have been largely discredited, and in most recurrent episodes of NA-AION, the cause remains unidentified. ${ }^{6}$

In most cases of primary NA-AION, a single definitive cause cannot be identified. Despite this fact, it is incumbent upon eye-care providers, in concert with other members of the patient's healthcare team, to detect predisposing and precipitating risk factors, some of which may be mitigated. By doing so, ocular morbidity can be minimized by lowering the chance of contralateral eye involvement and ipsilateral recurrence. Additionally, better management of underlying systemic disease can diminish the risk of systemic mortality. 
In this case, the main focus of care following the patient's NA-AION was discussing and altering any potential predisposing and precipitating systemic risk factors. However, there is some controversy surrounding the treatment of NA-AION with systemic corticosteroids. A large, prospective study of 696 eyes found that, in eyes with initial visual acuity of $20 / 70$ or worse, seen within 2 weeks of onset, visual acuity improved in $70 \%$ of the treated group compared to $41 \%$ of the untreated group..$^{29}$ Others refute the validity of this study, citing several inconsistencies. ${ }^{30}$ The presenting visual acuity in the right eye in this case does not fall within the criteria of the aforementioned study, and thus systemic corticosteroids were not considered.

Visual acuity and visual field loss are common consequences of NA-AION, but are not the only symptoms that patients struggle with following ischemic damage to the ONH. Contrast sensitivity loss and photophobia are debilitating problems following NA-AION that can be just as impactful as loss of visual acuity. The world is composed of infinite shades, and poor contrast sensitivity prevents patients from deciphering these subtle changes in their visual world. Patients will often complain that there is not enough or too much light in the environment, which can cause problems with glow and contour detection of objects. ${ }^{31}$ Additionally, an association between contrast sensitivity and functional independence has been shown. ${ }^{31,32}$ Thus, it is important for vision rehabilitation specialists to address four main visual symptoms after NA-AION, including visual acuity, contrast, photophobia, and visual field loss. $\bullet$

\section{REFERENCES}

1. Ischemic Optic Neuropathy Decompression Trial Research Group. Optic nerve decompression surgery for nonarteritic anterior ischemic optic neuropathy (NAION) is not effective and may be harmful. JAMA 1995;273:625-32.

2. Hayreh SS, Zimmerman MB. Nonarteritic anterior ischemic optic neuropathy: natural history of visual outcome. Ophthalmology 2008;115:298-305.

3. Hayreh SS. Management of ischemic optic neuropathies. Ind J Ophthalmol 2011;59:123-6.

4. Hayreh SS. Ischemic optic neuropathies-where are we now? Graefes Arch Clin Exp Ophthalmol 2013;251:1873-84.

5. Beri M, Klugman MR, Kohler JA, Hayreh SS. Anterior ischemic optic neuropathy. VII. Incidence of bilaterality and various influencing factors. Ophthalmology 1987;94:1020-8.

6. Hayreh SS, Podhajsky PA, Zimmerman B. Ipsilateral recurrence of non-arteritic anterior ischemic optic neuropathy. Am J Ophthalmol 2001;132:734-42.

7. Miller N, Subramanian P, Patel V. Walsh \& Hoyt's clinical neuroophthalmology: The essentials, vol. 3. Philadelphia, PA: Wolters Kluwer, 2016:145-160.

8. Hayreh SS. Ischemic optic neuropathy. Prog Retin Eye Res 2009 Jan;28(1):34-62.

9. SS. Blood supply of the optic nerve head and its role in optic atrophy, glaucoma, and oedema of the optic disc. Br J Ophthalmol 1969;53:721-48.

10. Hayreh SS. The 1994 Von Sallman Lecture. The optic nerve head circulation in health and disease. Exp Eye Res 1995;61:259-72.

11. Wright Mayes E, Cole ED, Dang S, et al. Optical coherence tomography angiography in nonarteritic anterior ischemic optic neuropathy. J Neuroophthalmol 2017 Dec;37(4):358-64.

12. Liu CH, Kao LY, Sun MH, Wu WC, Chen HS. Retinal vessel density in optical coherence tomography angiography in optic atrophy after nonarteritic anterior ischemic optic neuropathy. J Ophthalmol 2017;2017:9632647.

13. Hayreh SS. Acute ischemic disorders of the optic nerve: pathogenesis, clinical manifestations and management. Ophthalmol Clin North Am 1996;9:407-42.

14. Hayreh SS. Blood flow in the optic nerve head and factors that may influence it. Prog Retin Eye Res 2001;20:595-624.

15. Hayreh SS, Zimmerman MB. Nonarteritic anterior ischemic optic neuropathy: clinical characteristics in diabetic patients versus nondiabetic patients. Ophthalmology 2008;115(10):1818-25.

16. Jacobson DM, Vierkant RA, Belongia EA. Nonarteritic anterior ischemic optic neuropathy. A case-control study of potential risk factors. Arch Ophthalmol 1997;115:1403-07.

17. Mojon DS, Hedges 3rd TR, Ehrenberg B, et al. Association between sleep apnea syndrome and nonarteritic anterior ischemic optic neuropathy. Arch Ophthalmol 2002;120:601-5.
18. Hayreh SS, Zimmerman MB. Nonarteritic anterior ischemic optic neuropathy: refractive error and its relationship to cup/disc ratio. Ophthalmology 2008;115: 2275-81.

19. Hayreh SS. Anterior ischemic optic neuropathy. IV. Occurrence after cataract extraction. Arch. Ophthalmol 1980;98:1410-6.

20. SS. In vivo choroidal circulation and its watershed zones. Eye 1990;4:273-89.

21. Hayreh SS. Role of nocturnal arterial hypotension in the development of ocular manifestations of systemic arterial hypertension. Curr Opin Ophthalmol 1999;10(6):474-82.

22. SS, Podhajsky P, Zimmerman MB. Role of nocturnal arterial hypotension in optic nerve head ischemic disorders. Ophthalmologica 1999;213:76-96.

23. SS, Zimmerman MB, Podhajsky P, Alward WL. Nocturnal arterial hypotension and its role in optic nerve head and ocular ischemic disorders. Am J Ophthalmol 1994;117:603-24.

24. Hayreh SS, Podhajsky PA, Zimmerman B. Nonarteritic anterior ischemic optic neuropathy: time of onset of visual loss. Am J Ophthalmol 1997;124:641-7.

25. Newman NJ, Scherer R, Langenberg P, et al. The fellow eye in NAION: report from the ischemic optic neuropathy decompression trial follow-up study. Am J Ophthalmol 2008;134:317-28.

26. SS, Zimmerman MB. Optic disc edema in non-arteritic anterior ischemic optic neuropathy. Graefes Arch Clin Exp Ophthalmol 2007;245:1107-21.

27. Beck RW, Savino PJ, Schatz NJ, Smith CH, Sergott R. Anterior ischemic optic neuropathy: Recurrent episode in the same eye. $\mathrm{Br} \mathrm{J}$ Ophthalmol 1983;67:705-9.

28. Hamed LM, Purvin V, Rosenberg M. Recurrent anterior ischemic optic neuropathy in young adults. J Clin Neuroophthalmol 1988 Dec;8(4):239-48

29. Hayreh SS, Zimmerman MB. Non-arteritic anterior ischemic optic neuropathy: Role of systemic corticosteroid therapy. Graefes Arch Clin Exp Ophthalmol 2008;246:1029-46.

30. Atkins EJ, Bruce BB, Newman NJ, Biousse V. Treatment of nonarteritic anterior ischemic optic neuropathy. Surv Ophthalmol 2010;55(1):47-63.

31. Dos Santos NA, Andrade SM. Visual contrast sensitivity in patients with impairment of functional independence after stroke. BMC Neurol 2012 Sep 12;12:90.

32. Wolter M, Preda S. Visual deficits following stroke: maximizing participation in rehabilitation. Top Stroke Rehabil 2006 Summer;13(3):12-21. 